\title{
The influence of hydrodynamic factors, membrane surface properties and channel geometries on membrane performance and fouling mechanisms
}

\author{
Alexey Pervov ${ }^{1, *}$ \\ ${ }^{1}$ Moscow State University of Civil Engineering, Department of Water Supply and Water Disposal, \\ Yaroslavskoye sh. 26, 129337 Moscow, Russia
}

\begin{abstract}
Modern theoretical understanding of colloidal and suspended matter membrane fouling mechanisms are presented and discussed. Stateof-the-art simulation models of concentration polarization calculations for different channel conditions are described and influence of the fouling layers on the flux and rejection decrease are evaluated. Results of experimental investigations are presented that suggest a quantitative evaluation of fouling rates and membrane flux prognosis due to colloidal fouling with time. The influence of channel geometry on fouling is demonstrated and discussed. The main disadvantage of spiral wounded membrane modules which is attributed to the presence of a separation spacer mesh in the feed channel is discussed.
\end{abstract}

\section{Introduction}

The problem of fouling emerged at the beginning of industrial application of membranes to treat natural water for drinking and technical purposes. Theoretical and experimental investigations of membrane fouling processes reveal that processes of membrane transport, flow hydrodynamics, and chemical interactions that occur in membrane modules are very closely connected. Many modern research reports present results of membrane fouling investigations and develop fouling theories without accounting this relationship. Each process is very important to create a broad understanding of fouling.

Natural surface water contains suspended and colloidal matter and dissolved organics that have different molecular weight. Fouling mechanisms are different for different foulants. Suspended and colloidal matter can sediment on membrane surface, adhere to membrane, and/or coagulate. They form fouling layers that decrease membrane flux. Organic molecules adsorb on membrane surface or on foulant particles.

The first step to solving this problem is to understand that fouling and membrane performance deterioration was completely attributed to hydrodynamic factors (e.g., crossflow velocity, flux, etc.) and development of concentration polarization effect on membrane surface was due to uneven velocities distribution. Sorption and adhesive

* Corresponding author: ale-pervov@yandex.ru 
processes were not diligently accounted for. The main techniques to control fouling were based on creation of suitable flow conditions in membrane channels. Meanwhile, further experience demonstrated that hydrodynamic tools to control fouling have been exhausted. Other important factors to be taken into consideration are organic adsorption and the adhesion of particles and bacteria to membrane surface. Membrane material properties (e.g., charge, hydrophility, and adsorption characteristics) are decisive for fouling intensiveness and selection of these properties becomes an essential tool to control colloidal, organic and bacterial fouling. Low fouling membranes with a modified surface are developed and introduced into practice [1]. But it is not only flow hydrodynamics and surface properties that completely solve the problem.

Membrane channel configuration also plays important role in fouling. For a long time it was considered that spiral wound construction has high fouling propensities and requires very careful pretreatment. Currently, to remove colloidal and organic matter pretreatment uses ultrafiltration membranes tailored by tubular or capillary membrane channels that are considered more reliable than spiral wound configurations as they have lower hydraulic resistance and less membrane backwashing [2].

Spiral wound configuration is considered very efficient because of its compact design and high membrane surface per unit volume of the module. The main disadvantages of spiral wound modules are attributed to the presence of separation spacer mesh in the feed channel as it traps fouling particles and increases crossflow resistance [3, 4]. Elimination of said spacer mesh from the feed channels could be a decisive step in solving the fouling problem and developing efficient surface water and wastewater treatment techniques.

\section{Simulation models and analysis of colloidal fouling influence on reverse osmosis (RO) membrane mechanisms}

Prediction of product flow decrease due to membrane fouling remains one of the most important problems of RO process design and operation. Such prognostic techniques are based on the understanding of fouling mechanisms by authors who developed them.

A review of existing fouling theories demonstrated different views on the process. Filtration theory is developed and discussed in many publications presented by G. Belfort [5-9]. The described mechanism follows "cake filtration" mechanism. Belfort presents a filtration theory as a main tool to describe fouling of microfiltration membranes both in a "dead end" and cross-flow modes [7] that correspond to flow conditions in RO modules.

In [8], Belfort and co-authors developed a filtration theory that accounts for trajectories of particles in the cross-flow mode depending on their mass and velocity. As it was shown in [8], filtration theory often predicts stronger product flow decrease than was observed during experiments. Therefore, conventionally used filtration mechanism was improved in respect to determination of force that influences the particles in the flow. Calculations were made for laminar flow conditions with a parabolic distribution of velocities in the channel. The particle near membrane surface is influenced by convective and back diffusion forces and also by the lifting force that is formed due to difference of various layers velocities.

Theory of stability of colloidal solutions at membrane surface, as described by Brunelle in [10] and later developed in [11], combines concentration polarization effect with coagulation of particles on membrane surface. Coagulation occurs due to the increase of salt concentration in the boundary layer and the loss of colloidal solution stability.

Gel formation theory, or gel-polarization theory [12], describes coagulation or gel formation in high molecular organic solutions at membrane surface due to the concentration polarization effect. According to this theory, gel is formed after the maximum concentration value is reached near membrane surface. 
Colloidal fouling model based on adsorption mechanisms is presented by Jackson and Landolt [13] in respect to iron dioxide colloidal solutions. According to their suggested mechanism, membrane fouling occurs in two stages: nucleation and foulant growth. To describe foulant growth process, the authors use colloidal stability theory [10]. To describe and predict product flow decrease due to membrane fouling, cake filtration mechanism is widely used. The influence of accumulated fouling layers on membrane behavior is discussed below.

\section{The influence of fouling layers on RO membrane performance: product flow decrease and loss of rejection}

Different understandings also exist about the process of foulant influence on membrane performance. According to Kimura [14], accumulation of foulant on membrane surface leads to product flow decrease due to hydraulic resistance growth of the fouling layer. The decrease of product flow also decreases concentration polarization level, therefore membrane rejection value increases (Figure 1). This is not confirmed by the operational experience, as fouling always leads to loss of rejection.

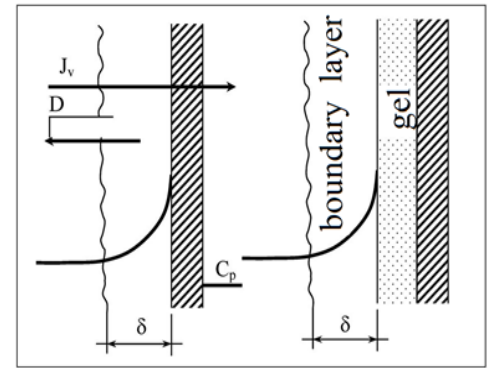

a)

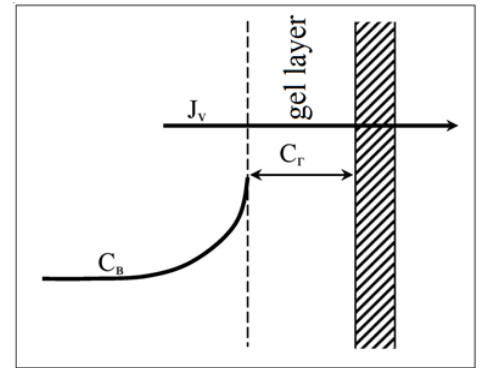

b)

Fig. 1. Increase of salt concentration near membrane surface due to layer formation: Kimura model (a) and gel formation model (b).

In [8], Belfort claims that rejection decrease (product quality deterioration) is connected with decrease of diffusion rate from membrane surface to the bulk solution due to the presence of the porous foulant layer on membrane surface. According to Merten's model, fouling layer thickness increase membrane boundary layer (where concentration polarization occurs), as shown in Figure 2. Therefore, reduction of membrane flux can be explained not by the increase of flow resistance (transmembrane pressure), but by the increase of concentration polarization and decrease of active pressure value that is described as $(P-\pi)$, where $\pi$ is osmotic pressure of solution on membrane surface.

The increase of flow resistance in membrane channels can significantly influence membrane flux as active pressure value decrease through the module length. It is well known that this resistance (called also "delta pressure") can reach high values, producing a "telescoping effect" of spiral wound membranes. Mechanism of flow resistance increase is greatly attributed to membrane channel configuration. Autopsies of fouled spiral wound membranes reveal that spacer mesh, mainly the mesh bundles are covered by foulant more than other membrane surfaces, trapping foulant particles [15]. Thus, flux reduction is attributed to the concentration polarization growth and active pressure value loss.

Hydraulic resistance of the fouling layer obviously does not influence RO membrane flux rate. Membrane autopsies show that fouling layers have very soft structure and its hydraulic resistance is negligible as compared to membrane resistance. Thus, calculations 
of fouling layers flow resistance do not have practical significance. In practical observation, flux decrease is always accompanied by an increase of product TDS. This drop of product flux due to the increase of foulant resistance does not explain the decrease of rejection values.
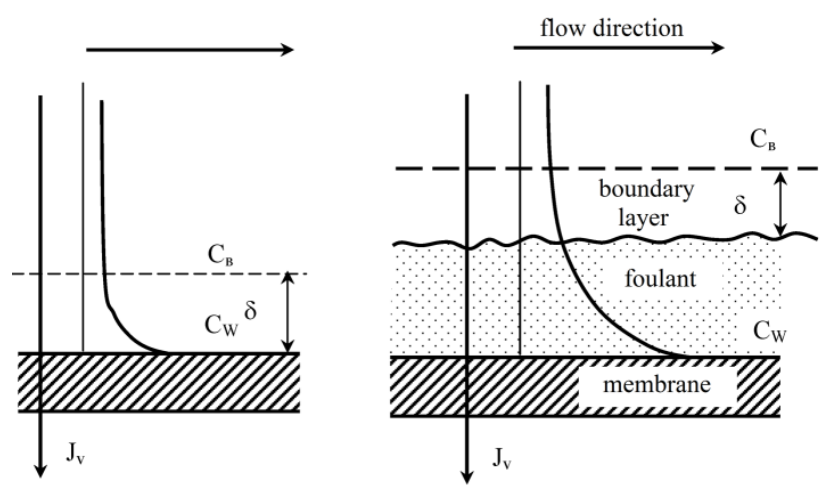

Fig. 2. Concentration profile near RO membrane surface in turbulent flow with fouling and without fouling layer influence.

Concentration polarization is thus recognized as the main reason of product flux decrease, and the thickness of the fouling layer is recognized as the main factor that affects membrane performance. When concentration polarization level is negligible (for the cases where tangential flow is high), the thickness of boundary layer is equal to the thickness of the fouling layer, and concentration polarization level in the fouled membrane channel is determined according to equation (1):

$$
C_{w} / C_{b}=\frac{\exp (v \delta / D)}{R+(1-R) \exp (v \delta / D)},
$$

where $C_{w}, C_{b}$ - salt concentration at the membrane wall and in bulk solution, $D$ - diffusion coefficient, $R$ - salt rejection o the membrane $\left(R=\left(C_{b}-C_{p}\right) / C_{b}, C_{p}\right.$ - salt concentration in permeate), $v$ - convective velocity, $\delta$ - thickness of boundary layer

The model that describes influence of the fouling layer on membrane rejection used the Nernst model equations. According to this model, a thin boundary layer exists at membrane surface (a stagnant film with a thickness $\delta$ ). Dissolved salt transport in this case is limited by molecular mechanism - diffusion that is determined by coefficient $D$. When fouling layer is formed on membrane surface, the thickness of boundary layer is increased by the thickness of the fouling layer, and salt transport through the layer from membrane to the bulk flow is limited as compared to the case where is no foulant (Figure 3).

Density of layer's structure can also influence membrane performance. Loose layer structure creates a lower thickness of the boundary layer than does a firm, dense structure.

Under laminar flow conditions a special velocity value profile is formed along with concentration profile in the boundary layer [16]. The concentration values profile is formed due to convection and diffusion. Concentration profile is formed even at the channel inlet. If the fouling layer is formed on membrane surface, concentration gradient is formed in the channel above this layer. The velocities' profile does not influence salt transport within fouling layer thickness. At the end of the channel, a new concentration gradient is built-up, which depends on velocities' profile in the bulk flow and within the fouling layer thickness, as shown on Figure 3. 


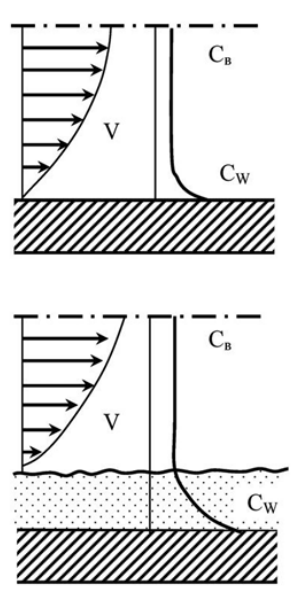

a)
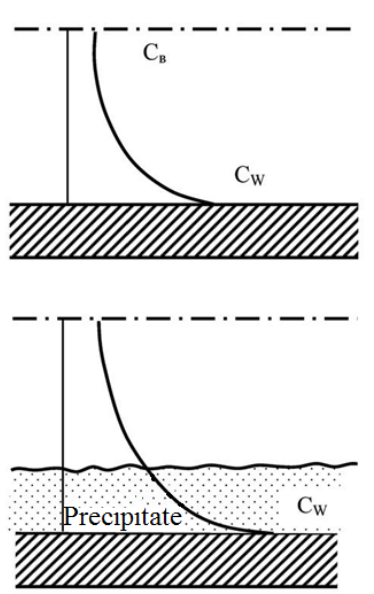

b)

Fig. 3. Development of concentration polarization with fouling under laminar flow conditions: at the channel inlet (a) and in the end of the channel (b).

\section{Colloidal and organic fouling: the influence of "shear" forces}

Cake filtration model is widely used to describe membrane fouling by suspended particles when water is filtered through microfiltration or ultrafiltration membrane in "dead-end" mode. As was already mentioned in [14], this model does not efficiently describe fouling layer formation under "cross-flow" conditions. In the "cross-flow" mode after the fouling layer is formed, the subsequent fouling does not occur due to "tangential" flow shear forces.

When surface water is treated with membranes, suspended particles, colloidal particles, and large organic molecules (e.g., humic acids) foul membrane surfaces. Colloidal particles differ by size and their diffusion characteristics vary. In the beginning of the process, a fouling layer forms on membrane surface and product flux decreases. The foulant growth stops after product flux decreases and concentration polarization level is reduced. Thus, cake filtration theory also can be used to describe fouling under "cross-flow" conditions. According to "cross-flow" model, fouling process consists of two steps:

1) accumulation of the fouling layer that cannot be sheared-off;

2) shearing rate and fouling rates reach equilibrium.

Research specialists often share their experience - that this equilibrium is reached usually after membrane unit is completely fouled-up and product flux has significantly been decreased [14]. Many technological tools to control colloidal fouling are recommended based on hydrodynamic understanding of the problem: to use flocculation to increase particle size and thus decrease fouling layer resistance; to increase "cross-flow" velocities and "shear-off" effect, to apply backwashes (for UF applications) and membrane flushing.

Fouling control by cross-flow velocities requires development of the "back transport" models. There are three models that describe shearing mechanisms: Brown's diffusion model for low molecular organics, "shear" diffusion for macromolecules, and migration model for colloids. These "back transport" models provide different evaluation of fouling potential for colloidal particles and humic substances. For small particle size and for low molecular weight substance, Brown's diffusion coefficient value is higher and "shear" diffusion coefficient value is lower than for large particles and high molecular organics. When organic solution is separated, concentration of organics in the boundary layer increases. Large organic molecules with smaller diffusion abilities are more likely to form fouling layer due to low solubility and higher concentration polarization level [17]. 
Product flux decrease when organic solution is separated is attributed to gel formation and sorption processes that occur between organic molecules and membrane material [18] and described by equation (2):

$$
J=\frac{P}{\mu\left(R_{m}+R_{a d s 1}+R_{a d s 2}\right)},
$$

where: $P$ - pressure drop through membrane; $R_{a d s l}$ - flow resistance of the gel layer plus additional resistance of adsorbed organic matter; $R_{a d s 2}$ - additional resistance attributed to interactions of adsorbed organics and dissolved organics.

Formation of gel fouling layer is described by the following equation (that is modified equation 2):

$$
\frac{P}{\mu J}=R_{o}+R_{c}=K_{c} \frac{V}{A}+\frac{P}{\mu J_{o}},
$$

where: $J_{0}$ - initial membrane flux velocity, $R_{0}$ - membrane resistance; $V$ - permeate volume; $A$-membrane surface; $K_{c}$ - a unit gel resistance coefficient; $R_{c}$-gel resistance.

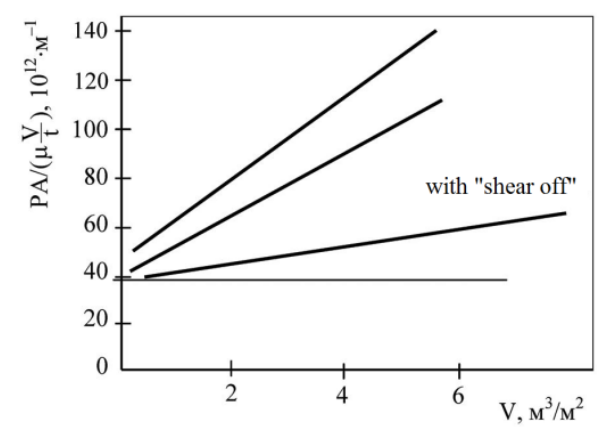

Fig. 4. Dependence of $P A /(\mu V / t)$ value on filtered volume for various cross-flow velocities [19]. 

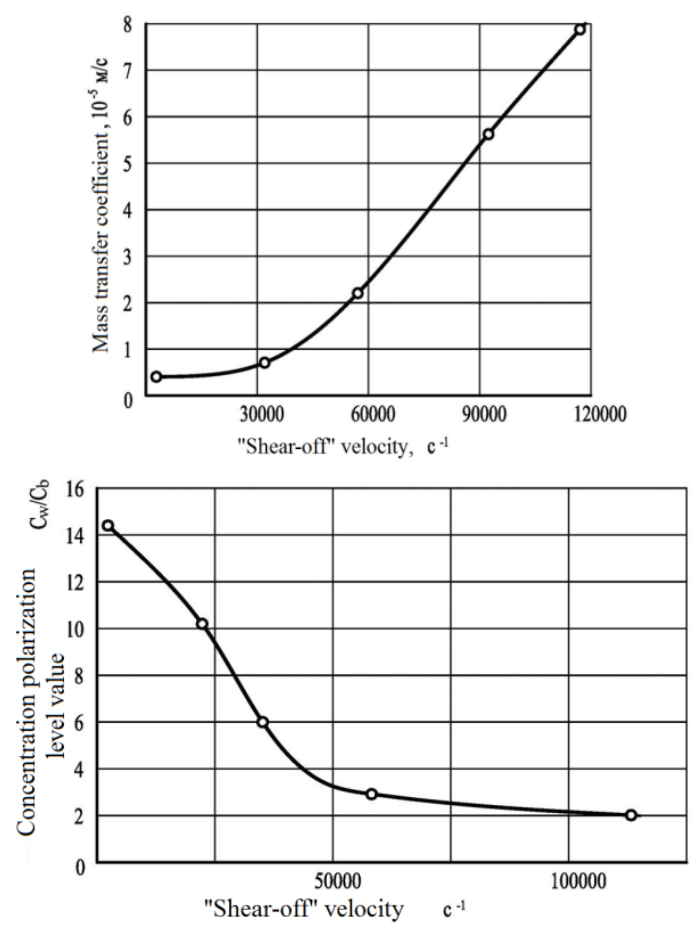

a)

b)

Fig. 5. The influence of "shear-off" velocity values on mass transfer coefficient and concentration polarization effect [19].

Figure 4 shows dependence of the $P A /(\mu V / t)$ value on the volume $V$ of water passed through membrane filter $\left(V / t=J_{t}\right.$, where $J_{t}$-membrane flux at time $\left.t\right)$. The change of slope can be explained by particle "shear-off" effect, decrease of their concentration, and gel layer resistance [19]. Figure 5 demonstrate the influence of cross-flow velocity values on concentration polarization level and mass transfer coefficient value.

\section{Increase of hydraulic resistance in membrane channels during operation}

The main disadvantages of spiral wound modules are attributed to the presence of separation spacer mesh in the feed channel as it traps fouling particles and increase a crossflow channel resistance $[4,20]$. The places (spots) where mesh contacts the membrane surface provide "dead" areas without cross-flow, resulting in high concentration increase at the membrane surface that initiates formation of colloids and crystals of sparingly soluble salts.

When water is filtered through a sand bed, suspended and colloidal particles adhere to the sand particles and sediment in the pore volume forming "bridges" in pore channels that increase hydraulic resistance for the flow. Experimentally obtained relationships of hydraulic resistance versus time, flow and suspended matter concentrations demonstrate similar process for spiral wound membrane channel and sand filter bed (Figure 6). 


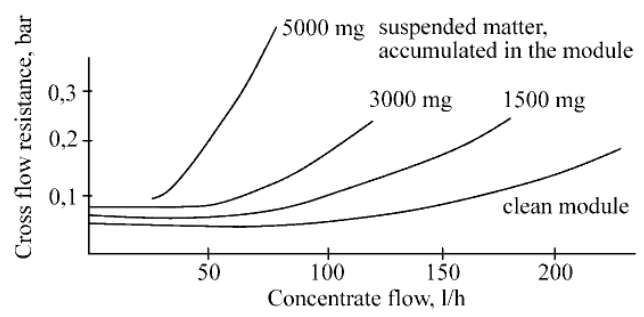

a)

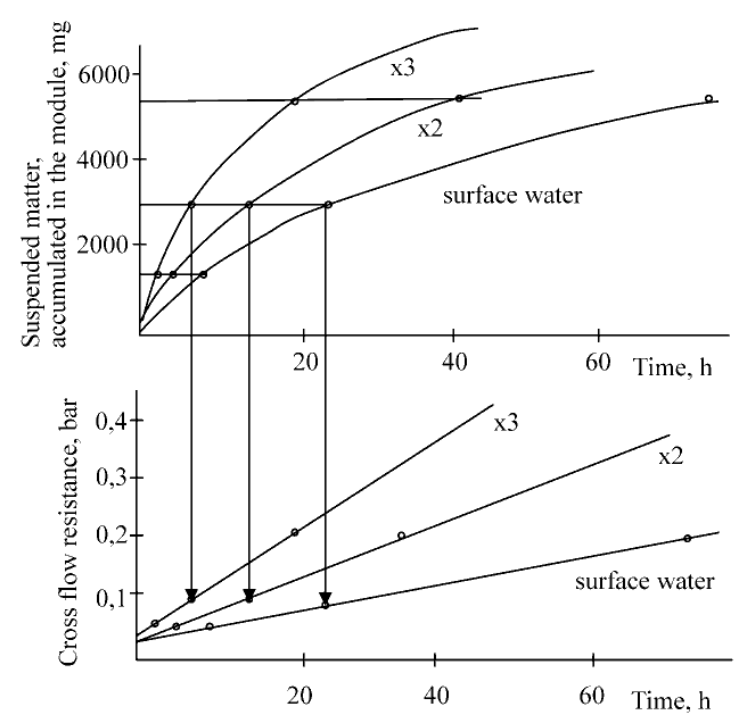

b)

Fig. 6. Example of flow resistance increase in spiral wound membrane module.

Figure 6 (a) shows the increase of cross-flow resistance versus cross-flow value at different stages of fouling (for different amounts of foulant, accumulated in the spiral wound module 1812 standard). Determination of fouling rate values at different stages of fouling (corresponding to different amounts of accumulated foulant) enabled us to predict amount of foulant sedimented on membrane surface depending on operation time and feedwater composition. Prognosis of foulant accumulation is demonstrated in Figure 6 (b), where feed water from the river was concentrated by RO twice and by three times and these solutions were tested. Based on the data presented in Figure 6 (b), each foulant amount corresponds to the operation time and to hydraulic resistance value (Figure 6 (c)).

\section{Conclusions}

Colloidal fouling rate values are very dependent on the cross-flow velocities: the higher cross-flow velocity, the less is foulant accumulates on membrane surface. Mechanism of suspended and colloidal fouling is mainly attributed to particle adhesion on membrane surface due to convective flow. Colloidal fouling varies depending on membrane properties such as surface charge and hydrophility. Channel geometry has decisive influence on fouling process. During foulant accumulation in spiral wound modules, cross-flow resistance grows. The product flux drop is directly dependent on hydraulic resistance increase as active pressure above membrane surface in the channel decreases. 
Future work will show that development of a new module with "open channel" with low cross-flow resistance enables us to suggest a reliable membrane water treatment technique that does not require pretreatment due to efficient channel configuration, modified membrane material and hydrodynamic mode.

\section{References}

1. J. Gilron, S. Belfer, P. Vaisanen, M. Nystrom, Desalination 140, 167-179 (2001)

2. X. Shi, G. Tal, N.P. Hankins, V. Gitis, J. of Water Process Eng. 1, 121-138 (2014)

3. C.P. Koutsou, A.J. Karabelas, J. of Membrane Sci. 488, 129-142 (2015)

4. A.H. Haidari, S.G.J. Heijman, W.G.J. Meer, Water Res. 106, 232-241 (2016)

5. G. Belfort, G. Alexandrowisz, B. Marx, Desalination 28, 13-30 (1979)

6. G. Belfort G., Desalination 34, 159-169 (1980)

7. R.H. Reed, G. Belfort, Water Sci. Tech. 14, 499-522 (1982)

8. G. Belfort, R.J. Weigand, R.I. Mahar, Reverse Osmosis and Ultrafiltration Symp. 188th Meet. Amer. Chem. Soc. (Philadelphia, Washington, 1985).

9. G. Green, G. Belfort, Desalination 35, 129-147 (1980)

10. M.T. Brunnele, Desalination 32, 127-135 (1980)

11. J.W. Kaakinen, C.D. Moody, Reverse Osmosis and Ultrafiltration Symp. 188th Meet. Amer. Chem. Soc. (Philadelphia, Washington, 1985).

12. S.-I. Nakao, T. Nomura, S. Kimura, AICHE Journal 25, No4, 615 (1979)

13. J.M. Jackson, D. Landolt, Desalination 12, 361-378 (1973)

14. D.E. Potts, R. Alert, S. Wang, Desalination 36, 235-264 (1981)

15. A.I. Shafer, A.G. Fane, T.D. Waite, Desalination 131, 215-224 (2000)

16. J. Cho, G. Amy, Y. Yoon, J. Sohn, Desalination 142, 245-255 (2002)

17. J.G. Grespo, K.W. Buddeken (ed.) Membrane processes in separation and purification (NATO ASI Series, 1994)

18. T.L. Champlin, Proc. of the Conf. on Membranes in Drinking and Industrial Water Production (Paris, France, 2000)

19. R. Bian, K. Yamamoto, Y. Watanabe, Proc. of the Conf. on Membranes in Drinking and Industrial Water Production (Paris, France, 2000)

20. A. Pervov, A. Andrianov, Desalination and Water Treatment 35, 2-9 (2011) 